\title{
ARTICLE
}

\section{Scintillation Properties of Composite Stilbene Crystal for Neutron Detection}

\author{
Seung Kyu LEE ${ }^{1}$, Yun Ho CHO ${ }^{1}$, Byoung Hwi KANG ${ }^{1}$, Woo Gyo LEE ${ }^{1}$, Jong Kyung KIM ${ }^{1}$, \\ Gi Dong KIM ${ }^{2}$, Nikolai Z. Galunov ${ }^{3}$, and Yong Kyun KIM $^{* 1}$ \\ ${ }^{1}$ Department of Nuclear Engineering, Hanyang University, Seoul, 133-791, Korea \\ ${ }^{2}$ Korea Institute of Geoscience and Mineral Resources, Daejeon, 305-350, Korea \\ ${ }^{3}$ Istitute for Scintillation Materials of Scientific Technology Center "Institute of Single Crystals" of NASU of Ukraine
}

\begin{abstract}
The large area stilbene scintillator was proposed for use as neutron-flux monitors in measuring the characteristics of neutron radiation in the Korea Superconducting Tokamak Advanced Research (KSTAR). A composite stilbene scintillator with crystal $\Phi 50 \mathrm{~mm} \times 12 \mathrm{~mm}$ was specially developed for fast neutron detection. The detector response measurements have been performed by using gamma, alpha and neutron radiation source and compared with the commercial single stilbene.
\end{abstract}

KEYWORDS: stilbene, neutron monitor, KSTAR, composite stilbene scintillators, light yield

\section{Introduction}

Nowadays, the measurement of fast neutron is the most crucial issues in the facilities, such as particle accelerator, generation IV nuclear reactor (GEN-IV), nuclear fusion reactor (KSTAR in Korea) and so on. Therefore it is very important to measure the fast neutron precisely against the background gamma-rays. The most common method of fast neutron detection is based on elastic scattering of neutrons. One of the easiest ways to detect recoil protons produced by fast neutrons is through the organic scintillators, such as anthracene, stilbene crystals and so on. Fast neutrons in the material like an organic scintillator produce recoil protons by $(n, p)$ reaction, and the highest energy of a recoil proton that happen in time equals the energy of neutron. ${ }^{1)}$

Stilbene is one of the representative organic scintillator for the detection of fast neutron. The light output of stilbene is as low as about $30 \%$ of $\mathrm{NaI}(\mathrm{Tl})$, whereas it has good advantage to compare light output that is created by charged particles and electrons. For this reason, stilbene had been used widely to measure neutrons discriminating the background of accompanying gamma-rays. However, since stilbene single crystals are difficult and expensive to obtain in large sizes greater than a few centimeters in dimension and are also subject to damage from thermal and mechanical shock, it is common to use a plastic or liquid scintillator for the fast neutron applications. ${ }^{2)}$ On the other hand, these types of scintillators have problems. They have lower light output than single crystal and liquid scintillators are toxic and flammable. Therefore, the new types of organic scintillators with polycrystal structure have been studied in several research institutes. This new type of organic scintillation detector is composite scintillator based on grains of stilbene crystals. $^{1-2)}$

Corresponding Author, Tel No: +82-2-2220-2354, Fax No:

+82-2-2296-2354, E-Mail : ykkim4@hanyang.ac.kr

\section{Scintillator sample preparation \\ 1. Stilbene single crystal}

The new scintillation materials were obtained on the base of perfect stilbene single crystal's grains. The grain of stilbene single crystal was made by crushing the stilbene single crystal at the low temperature of liquid nitrogen. Stilbene crystals were grown from Bridgeman-Stockbarger method, ${ }^{3-4)}$ whose typical physical properties are presented in Table 1. The commercial stilbene single crystal with $9 \mathrm{~mm}$ thickness and $50 \mathrm{~mm}$ in diameter was used in the experiment, as shown in Fig. 1 a).

Table 1 General properties of stilbene crystal. ${ }^{5)}$

\begin{tabular}{cc}
\hline Property & Value \\
\hline Light output $(\%$, Anthracene) & 50 \\
Density $\left(\mathrm{g} / \mathrm{cm}^{3}\right)$ & 1.16 \\
Decay Time $(\mathrm{ns})$ & 4.5 \\
Peak Emission $(\mathrm{nm})$ & 410 \\
No. of H atoms/ No. of C atoms & 0.858 \\
\hline
\end{tabular}

\section{Composite Stilbene Scintillator}

The 'composite stilbene scintillator' was manufactured with stilbene crystal grains into organosilicone base. We chose a grain size range from 1.5 to $2.5 \mathrm{~mm}$. The $11 \mathrm{~mm}$ thickness and $50 \mathrm{~mm}$ in diameter composite stilbene scintillator was fabricated and used in the experiment. The method of preparation of the composite stilbene scintillator as the detector of fast neutrons includes the following steps.

First, a stilbene single crystal was grown by Bridgeman -Stockbarger method. Then, we obtained the stilbene crystal grains of this grown crystal by grinding at a low temperature with liquid nitrogen and choosing the stilbene crystal grain size range from 1.5 to $2.5 \mathrm{~mm}$. After above performance, these crystal grains stayed in dark place at least 24 hours to stabilize.

Second, we prepared the light guide housing which determine the size and shape. Prepared housing container 
size equalizes the composite stilbene scintillator with $12 \mathrm{~mm}$ thickness and $50 \mathrm{~mm}$ in diameter. And we mixed the crystal grains and the organosilicone base, sylgard-527 component $\mathrm{A}$ and $\mathrm{B}$. Then, the composition was located in the housing container.

Finally, this composite stilbene scintillator stayed in vacuum place during 30 minutes. And then, to polymerize of sylgard-527 optical glue, it stayed in dark place with pressure on the upside. Final density of this composite stilbene was $0.9486 \mathrm{~g} / \mathrm{cm}^{3}$. To improve a light collection, composite stilbene scintillator was coated by reflective material Tetratek(UK). ${ }^{6}$ Figure 1. b) shows the composite stilbene scintillator.

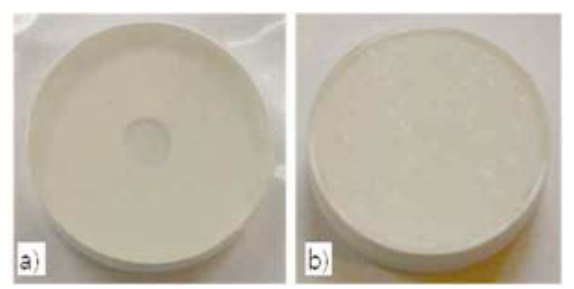

Fig. 1 Photography of the two type of organic scintillatiors: a) is a $\Phi 50 \mathrm{~mm}$ x $9 \mathrm{~mm}$ commercial stilbene single crystal covered by reflecting coating, b) is a $\Phi 50 \mathrm{~mm} \times 12 \mathrm{~mm}$ composite stilbene scintillator coated with a reflecting medium.

\section{Experiments and Results}

\section{Gamma-ray measurements}

A ${ }^{137} \mathrm{Cs} 0.662 \mathrm{MeV} \gamma$-ray source was used for the measurements of the light yield of $\Phi 50 \mathrm{~mm}$ x $12 \mathrm{~mm}$ composite stilbene scintillation detector. The $\Phi 50 \mathrm{~mm} \times 9$ mm stilbene single crystal was taken as the commercial stilbene scintillator. These scintillators were attached with a 2" 6654A EMI photomultiflier tube (PMT). And scintillators were coupled onto PMT's window with optical grease. Figure 2 shows a schematic diagram of the experimental setup for the radiation measurement. The high voltage of $1400 \mathrm{~V}$ was applied to the PMT.

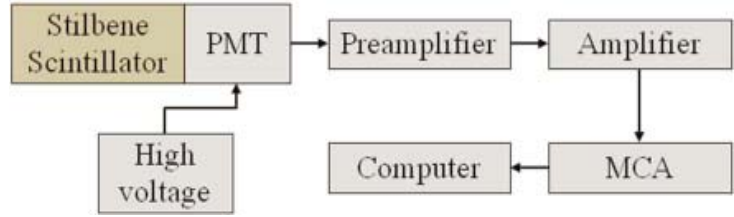

Fig. 2 Schematic diagram of the experimental setup

Before the main measurements, the energy calibration for gamma-radiation was performed. Figure $\mathbf{3}$ shows the calibration results for the commercial stilbene single crystal. For the given energy range, the response of a commercial stilbene single crystal was linear for the gamma-radiation. All the measurements were performed under same conditions. ${ }^{22} \mathrm{Na},{ }^{60} \mathrm{Co},{ }^{137} \mathrm{Cs},{ }^{152} \mathrm{Eu}$ gamma sources were used in energy calibration. Scintillation signals were proportional to the energy of the corresponding center of Compton edge which is defined as a middle point from peak to Compton edge as shown in Fig. 3.
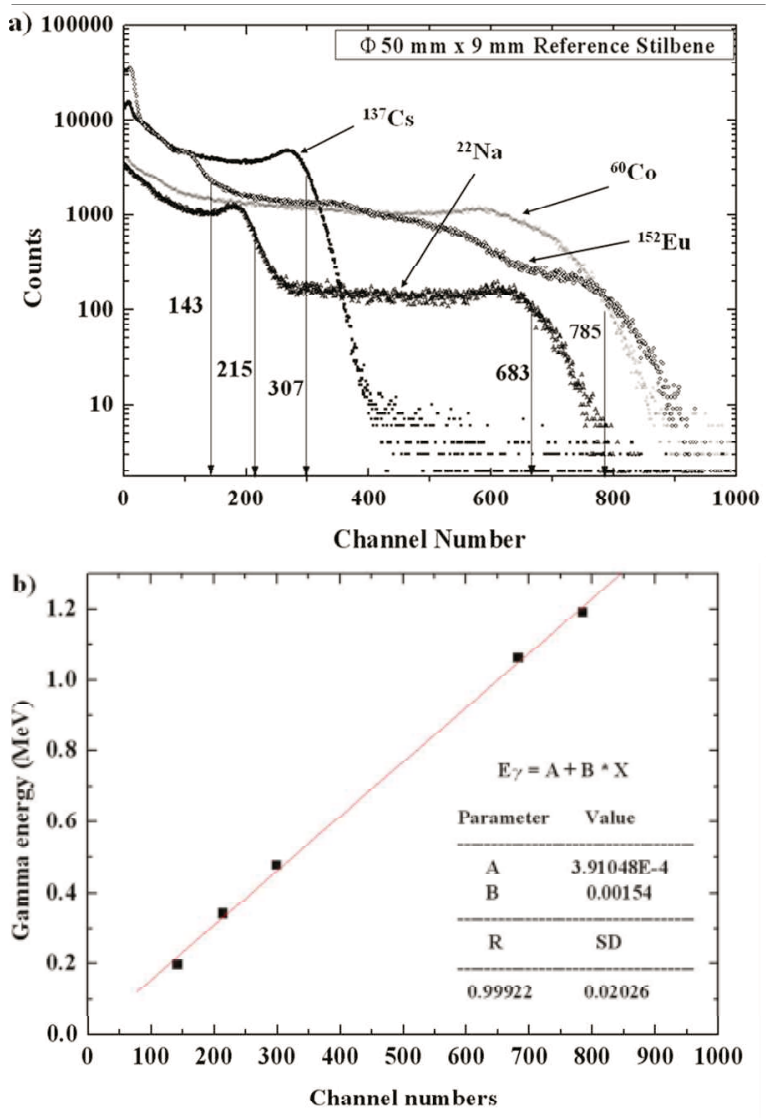

Fig. 3 a) Gamma-ray pulse height spectra for commercial stilbene single crystal to photon energy calibration, b) Result of calibration of amplitude for the measurement setup in the scale of gamma-radiation energies for a $\Phi 50 \mathrm{~mm} \mathrm{x} 9 \mathrm{~mm}$ commercial stilbene single crystal.

The $\mathrm{R}$ value is the square root of $\mathrm{R}^{2}$, which is a quantity that shows how good the fit is, and it can be computed by the following equation;

$$
R^{2}=\frac{\text { Explained variation }}{\text { Total variation }}=\frac{T S S-R S S}{T S S}
$$

where TSS is the total sum of square, and RSS is the residual sum of square. And $\mathrm{SD}$ is the root mean square of the error, or the standard deviation of the model.

The relative light yield of composite stilbene scintillator was calculated to the commercial stilbene scintillator's. The light yield for the commercial stilbene scintillator was measured by a method based on measuring of the intrinsic resolution of the photomultiplier. ${ }^{7)}$ The light yield of commercial stilbene for gamma-ray was equal to $1.5 \times 10^{4}$ photons/MeV. As shown in Fig. 4, center of Compton edge for the commercial stilbene single crystal was 307 channels, 
and the composite stilbene was 251 channels. The light yield of composite stilbene scintillator was possible to be gotten by the equation;

$$
L_{c o m}=\frac{C_{c o m}}{C_{r e f}} \times L_{r e f},
$$

where, $\mathrm{C}_{\mathrm{ref}}, \mathrm{C}_{\mathrm{com}}$, and $\mathrm{L}_{\mathrm{ref}}$ are channel number of compton edge for commercial stilbene single crystal, composite stilbene, and light yield of commercial stilbene single crystal.

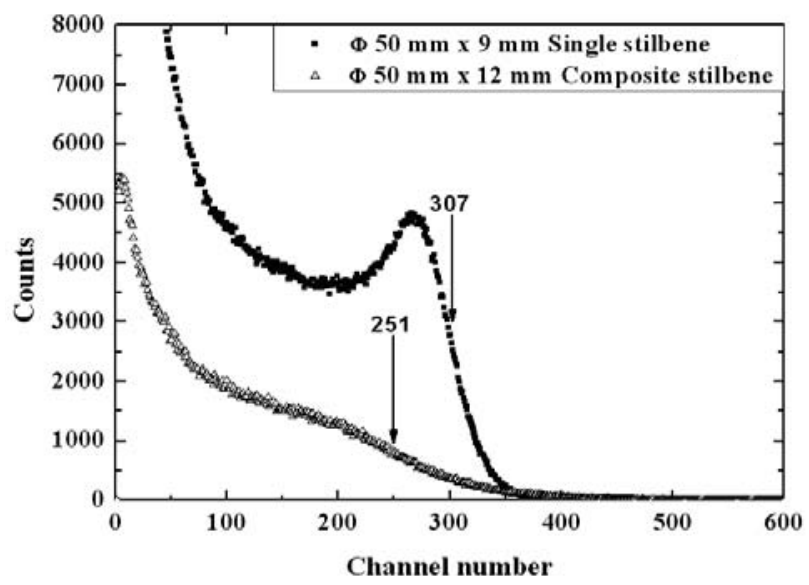

Fig. 4 The scintillation amplitude spectra for the stilbene single crystal and composite stilbene scintillators irradiated by gamma-ray from a ${ }^{137} \mathrm{Cs}$ Source

As a result, the light yield of composite stilbene scintillator for gamma-ray was equivalent to $1.23 \times 10^{4}$ photons $/ \mathrm{MeV}$. The relative light yield of composite stilbene comparing with commercial stilbene single crystal for gamma-radiation was about $81 \%$.

\section{Alpha particles measurements}

The results of measurements for the gamma-ray and the alpha particles give initial information about scintillation characteristic of the composite stilbene scintillator. A ${ }^{239} \mathrm{Pu}$ radiation source was used for alpha particle detection. The initial energy of ${ }^{239} \mathrm{Pu}$ alpha particle is $5.15 \mathrm{MeV}$. To improve energy resolution, $2 \mathrm{~mm}$ thickness collimator was used in the measurements. The collimator has numbers of $\Phi 1.5 \mathrm{~mm}$ holes. The alpha particle energy became about $4.97 \mathrm{MeV}$ after passing through air in the collimator holes.

Figure 5 shows the pulse height spectrum for $4.97 \mathrm{MeV}$ alpha particles of a ${ }^{239} \mathrm{Pu}$. As shown in Fig. 5, center of peak for the commercial stilbene single crystal was 205 channels and the composite stilbene was 142 channels. As a result, when the composite stilbene scintillator compared with commercial stilbene single crystal, the relative light yield of composite stilbene for alpha particles was about $70 \%$. The Table 2 shows the ratio of relative light yield for composite silbene scintillator compared with the commercial stilbene single crystal for gamma-ray and alpha particles.

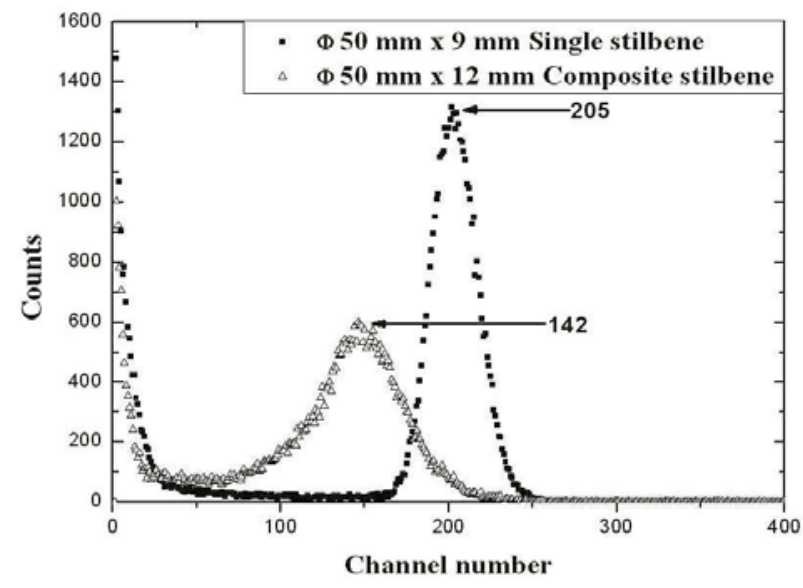

Fig. 5 The scintillation amplitude spectra for stilbene single crystal and composite stilbene scintillators irradiated $5.15 \mathrm{MeV}$ alpha particles from a ${ }^{239} \mathrm{Pu}$ source (after passing through air in the collimator holes, the energy became about $4.97 \mathrm{MeV}$ ).

Table 2 The relative light yield of the composite stilbene compared with commercial stilbene single crystal

\begin{tabular}{cc}
\hline Type of radiation & Light yield ratio (\%) \\
\hline Gamma-ray & 81 \\
Alpha particle & 70 \\
\hline
\end{tabular}

\section{Neutron Measurements}

$\mathrm{A}^{239} \mathrm{Pu}$-Be radionuclide source was used for fast neutrons. The special setup of discriminator was used to separate the neutrons and background gamma-rays, as shown in Fig. 6. It determines the difference in shape and intensity of slow component of scintillation pulse of organic crystals for the cases of excitation with different specific energy loss $\mathrm{dE} / \mathrm{dx} .^{8)}$

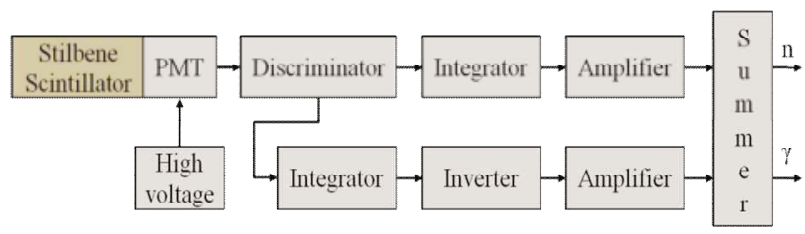

Fig. 6 Schematic diagram of the special setup of discriminator.

Figure 7 shows the recoil proton spectra generated by fast neutrons of ${ }^{239} \mathrm{Pu}-\mathrm{Be}$ radionuclide source in the commercial stilbene single crystal and in the composite stilbene scintillator. The relative detection efficiency of fast neutron was calculated by the total number of counts under the curve of a recoil proton spectrum. The detection efficiency is assumed as $100 \%$ for the commercial stilbene single crystal. The relative efficiency of fast neutron detection can be obtained by the following equation;

$$
\varepsilon=\frac{\sum C_{\text {com }}}{\sum C_{\text {ref }}}
$$




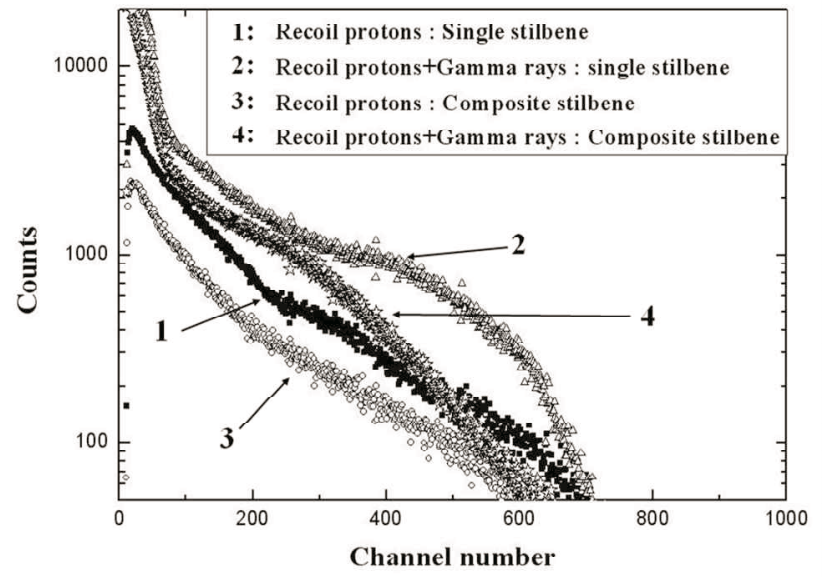

Fig. 7 The recoil proton spectra of a ${ }^{239} \mathrm{Pu}-\mathrm{Be}$ source for the reference stilbene single crystal and for the composite stilbene scintillator.

The total number of counts of fast neutron for the commercial stilbene single crystal was 526832 counts, and the composite stilbene scintillator was 281787 counts. The calculated value of the detection efficiency for composite stilbene scintillator was $53.5 \%$.

\section{Large Area Composite Stilbene Scintillator}

We had fabricated $\Phi 200 \mathrm{~mm}$ x $20 \mathrm{~mm}$ large size composite stilbenes using the previously mentioned method. And also, the housing of large area composite scintillator manufactured with aluminum, as shown in Fig. 8.

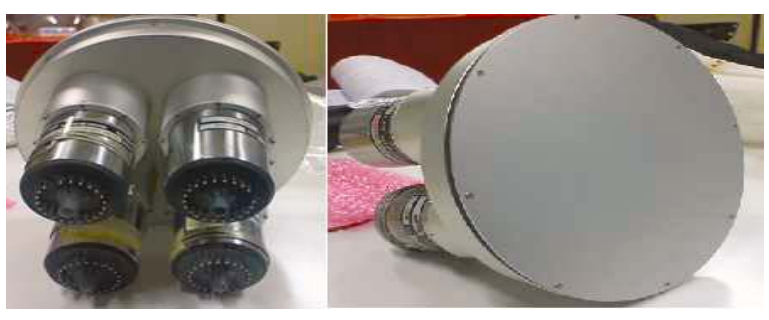

Fig. 8 The housing of large area composite stilbene scintillator

With further study about the large area composite stilbene scintillator, we will perform experiments to evaluate performance.

\section{Conclusion}

Composite stilbene scintillators based on grains of stilbene single crystals were manufactured and compared with stilbene single crystals. The composite stilbene scintillators can be manufactured as large area scintillators like liquid scintillators. These composite scintillators have advantages that they can be used without special housing, and do not have any toxicity. The property measurement result says that our composite stilbene scintillator has the scintillaion characteristics which are similar to single crystals for radiations, such as gamma-rays, alpha particles, and fast neutrons.

\section{Acknowledgment}

This work was supported by the nuclear R\&D program of MEST, Korea and also supported by the Ministry of Knowledge Economy (20090081806).

\section{References}

1) S. V. Budakovsky, N. Z. Galunov, B. V. Grinyov, N. L. Karavaeva, Jong Kyung Kim, Yong-Kyun Kim, N. V. pogorelova, O. A. Tarasenko, "Stilbene crystalline powder in polymer base as a new fast neutron detector" Radiation Measurements, 42 [4], 565 (2007).

2) G. F. Knoll, Radiation Detection and measurement, John Willey and Sons, New York, 220 (1999).

3) A. Arulchakkaravarthi, P. Santhanaraghavan, Rakesh Kumar, S. Muralithar, P. Ramasamy, T. Nagarajan, C. W. Lan, "Detection characteristics of vertical Bridgeman grown stilbene crystals for gamma rays using ${ }^{60} \mathrm{Co},{ }^{137} \mathrm{Cs}$ and ${ }^{22} \mathrm{Na}$ gamma ray sources" Materials Chemistry and Physics, 77[1], 77 (2002).

4) I. P. Krainov, N. Z. Galunov, and S. V. Budakovsky, "The influence of controlled electron seeking addition agents of the melt on stilbene single crystal structure and radioluminescence properties." Cryst. Res. Techn., 24, 193 (1989).

5) William R. Leo, Techniques for Nuclear and Particle Physics Experiments, A How-to Approach, Second Revised Edition, Springer-Verlag, New York, Berlin, Heidelberg, 159 (1994).

6) N. Z. Galunov, B. V. Grinyov, N. L. Karavaeva, Jong Kyung Kim, Yong Kyun Kim, O. A. Tarasenko, and E. V. Martynenko, "Development of new composite scintillation materials based on organic crystalline grains." IEEE Transactions on Nuclear Science, 56[3], 904 (2009)

7) E. Sysoeva, V. Tarasox, O. Zelenskaya, "Comparison of the methods for determination of scintillation light yield." Nuclear Instruments and Methods in Physics Research Section A, 486, 67 (2002)

8) Sergey V. Budakovsky, Nikolai Z. Galunov, Natalya L. Karavaeva, Jong Kyung Kim, Yong kyun Kim, Oleg A. Tarasenko, and Eugenia V. Martynenko, "New effective organic scintillators for fast neutron and short-range radiation detection" IEEE Transactions on Nuclear Science, 54[6], 2734 (2007) 